\title{
Benign cystic mesothelioma presenting as a solitary non-attached cyst in the pouch of Douglas
}

\author{
F. Israfilbayli • M. Shoukrey • A. Kadva
}

Received: 11 April 2007 / Accepted: 25 July 2007 / Published online: 25 September 2007

(C) Springer-Verlag 2007

\begin{abstract}
We present a case of a totally independent cyst floating freely in the pouch of Douglas. This cystic structure was incidentally found during laparoscopy in a 50-year-old woman whose ultrasound scan suggested an ovarian cyst or a possible hydrosalpinx. The cyst was removed and was histologically identified as a benign cystic mesothelioma, which in itself is an extremely rare tumour that presents in the form of solitary or multiple peritoneal cysts attached to serous membranes. In our case the cyst was completely unattached and in this regard represents the first report of a solitary unattached free floating benign cystic mesothelioma found in the pouch of Douglas.
\end{abstract}

Keywords Solitary $\cdot$ Cyst $\cdot$ Mesothelioma .

Benign · Douglas

\section{Introduction}

Benign cystic mesotheliomas are extremely rare mesenchymal neoplasms of unknown origin [1]. They were first described by Mennemeyer and Smith in 1979 [2]. We present an interesting, atypical, if not unique, presentation of such a tumour. During routine laparoscopy for what was

\footnotetext{
F. Israfilbayli $\cdot$ A. Kadva $(\bowtie)$

Department of Obstetrics and Gynaecology,

Colchester General Hospital,

Turner Road,

Colchester CO4 5JL, UK

e-mail: aban.kadva@essexrivers.nhs.uk

M. Shoukrey

Department of Obstetrics and Gynaecology,

Princess Alexandra Hospital,

Harlow CM20 1QX, UK
}

thought to be an ovarian cyst, an independent cystic structure was found roaming freely in the pouch of Douglas. This was later histologically confirmed to be a benign cystic mesothelioma.

\section{Case report}

A 50-year-old Caucasian woman with two previous pregnancies presented with a history of menorrhagia and peri-menopausal symptoms. She also experienced occasional frequency and urgency of micturition. The patient had been sterilised by laparoscopic clip sterilisation. Her medical history included epilepsy and depression for which she took sodium valproate and clonazepam, respectively. She also had a past history of deep venous thrombosis 9 years previously, following prolonged immobilization, and benign breast lumps.

In the gynaecology clinic, the abdominal examination was essentially unremarkable. The pelvic examination showed a small endo-cervical polyp. Bimanual examination revealed a small mobile uterus and no adnexal masses or tenderness were felt. Investigations included a full blood count which showed a haemoglobin level of $13.1 \mathrm{~g} / \mathrm{dL}$, and a midstream sample of urine was negative for microscopy and culture. A transvaginal ultrasound scan showed two subserosal fibroids, the largest measuring $7 \times 6 \times 5 \mathrm{~cm}$ arising from the right fundal wall and extending into the left adnexal region. The second fibroid was $2.4 \mathrm{~cm}$ arising from the left fundal wall. The endometrial thickness measured $21 \mathrm{~mm}$ with a possibility of two endometrial polyps. The right ovary was not enlarged. The left ovary appeared within normal limits with a closely related elongated $8 \times 4 \times 5 \mathrm{~cm}$ cystic area in the left adnexal region. The cystic area extended into the pouch of Douglas. It was 
predominantly cystic but there were two local solid areas indicating it may be an ovarian mass or a left hydrosalpinx (Fig. 1). The serum Ca125 was reported at $29 \mathrm{kU} / \mathrm{L}$ (normal range $0-35 \mathrm{kU} / \mathrm{L}$ ). The patient was listed for hysteroscopy and laparoscopy. A referral to the incontinence advisory team was made to assist with the urinary symptoms.

The patient was admitted for hysteroscopy and laparoscopy with attention to thromboprophylaxis. The hysteroscopy revealed a polypoid endometrial lining. There were no submucous fibroids or endometrial polyps. An endometrial curettage was performed and the cervical polyp was removed. The laparoscopy revealed a bulky uterus with two small fibroids arising from the left lateral and posterior uterine wall. Both tubes showed evidence of previous sterilisation and both ovaries were of normal appearance. A totally free-floating well-defined smooth-walled cystic structure, measuring approximately $8 \mathrm{~cm}$, was seen in the pouch of Douglas (Fig. 2). This cystic structure was easily extracted via a secondary port using an Endobag (Lina Medical). The cyst fluid was aspirated and sent for cytology (Fig. 3). The patient made an uneventful postoperative recovery, and was discharged home the following day.

The histology report of the cystic structure confirmed the diagnosis of a benign mesothelial cyst. The cyst fluid contained proteinaceous material and benign mesothelial cells, and no malignant cells were seen. The endometrial curettings were reported as showing proliferative changes with no hyperplasia or malignancy. The endocervical polyp was found to be benign.

\section{Discussion}

There are three different types of mesothelial tumours described in the literature that can present in the peritoneal cavity. These are ademantoid tumours, benign cystic mesothelioma, and malignant mesothelioma [3]. All three types are known to be very rare. Malignant mesothelioma

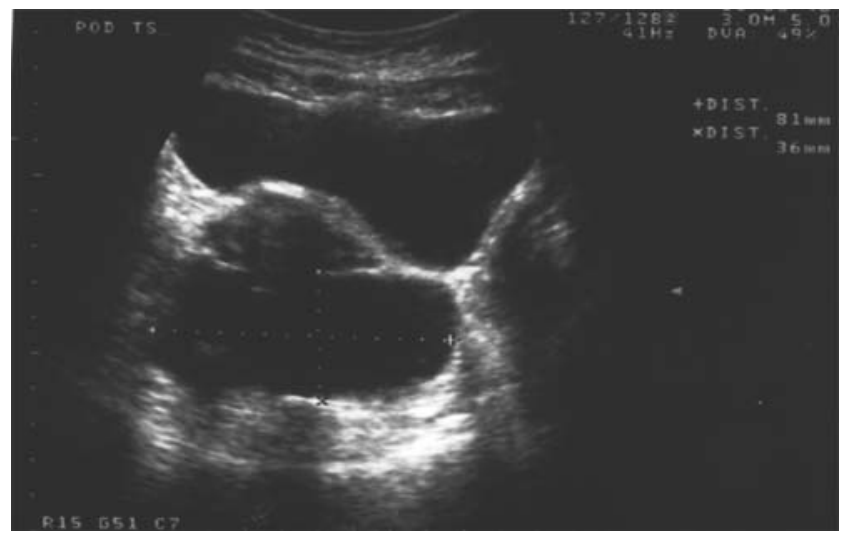

Fig. 1 Ultrasound scan showing an elongated $8 \times 4 \times 5 \mathrm{~cm}$ cystic area in the left adnexal region extending into the pouch of Douglas

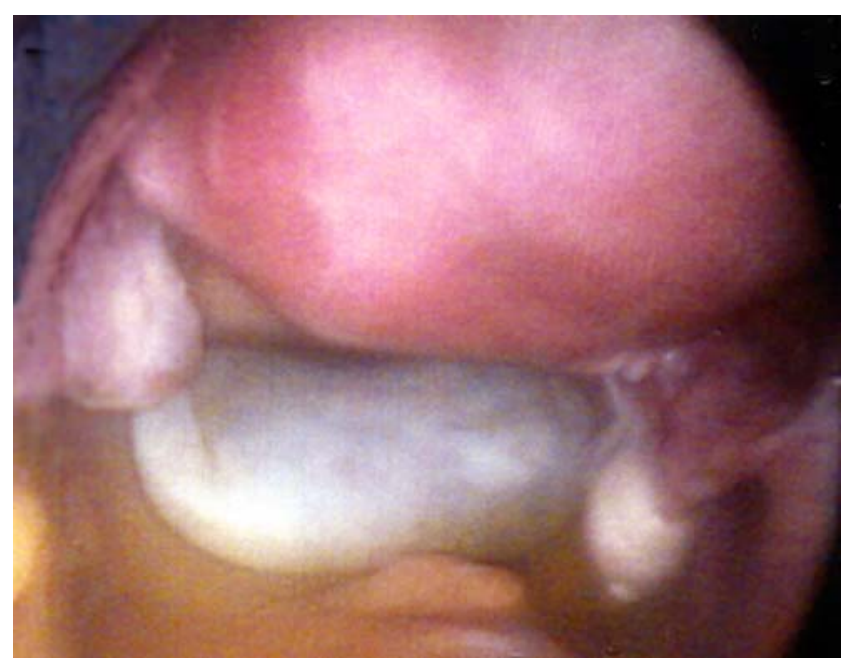

Fig. 2 Laparoscopy showing solitary mesothelial cyst floating freely in the pouch of Douglas

occurs at an incidence of 0.9 to 1.0 per million persons per year [4]. Ademantoid tumours are rare tumours occurring mostly in the genital tract. The type found in this study was a benign cystic mesothelioma (BCM). Only about 140 cases of BCM have ever been reported in the literature [3]. BCM occurs predominantly ( $84 \%$ of cases) in young or middle-aged women with the mean age being 37 years and 10 months $[5,6]$.

Mesothelial cells are typically flattened or cuboidal. In one third of patients, adenomatoid change or squamous metaplasia of the mesothelium is found. Unlike the malignant form of mesothelioma, multicystic mesothelioma has no association with asbestos exposure. This tumour seems to be more common in the Caucasian race with the previous surgery being one of the factors implicated in its pathogenesis [7].

A most common presentation is pain with or without an abdominal mass. In the majority of cases symptoms present for several months and only few are acute presentations. In

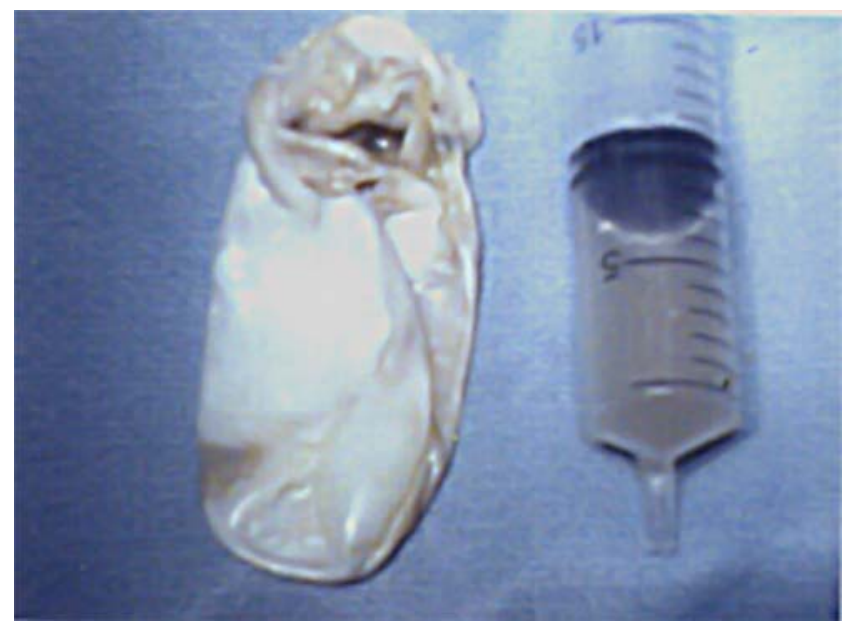

Fig. 3 Photo of the mesothelial cyst with aspirated fluid in a syringe 
our case, the patient presented with symptoms not related to the cyst and the existence of the latter was actually discovered by scan. Some rare associations are also mentioned in the literature such as mucinous cystadenoma, pseudomyxoma peritonei, leiomyomatosis peritonealis disseminate, and serous adenomatoid tumour of the uterus [8]. The tumour can be recurrent.

BCM usually presents as solitary or multilocular thinwalled cysts containing watery fluid [9]. When BCM affects the pelvic peritoneum, particularly the uterus, culde-sac, bladder, and rectum, it grows along the serosa as multiple translucent fluid-filled cysts [10]. We present the first case ever reported as an independent nonattached cyst in the pouch of Douglas. In fact, to the best of our knowledge, this is the first time a nonattached cyst has ever been found free in the pouch of Douglas regardless of its histological nature. We speculate that the cyst was attached to the serosa of the uterus, sigmoid, or rectum and then got detached.

The condition can be diagnosed with ultrasound, CT scan, or MRI imaging. It will, however, only be confirmed by electron microscopy and immunohistochemistry following surgery. The presence of long slender apical microvilli, abundant intracytoplasmic organelles, and filaments with hobnailed appearance of the cells are some of the distinguishing features of the mesothelial cells [7]. Immunohistochemical markers help differentiate between epithelioid mesotheliomas and serous carcinomas. The best positive markers for diagnosing mesotheliomas are D2-40, podoplanin, and calretinin. The best negative markers are Ber-EP4 and MOC-31 [11].

The treatment is usually in the form of surgical resection. Recurrence is possible and therefore follow-up after surgery is recommended. BCM is not chemosensitive or radiosensitive. No correlation exists between the extent of the tumor and the patient's survival [10].

We found the rare case when the mesothelioma was found distinct in the pouch of Douglas, not related or connected to any structure within the pelvis as it usually does. BCM are usually multicystic multiple, but in our case it was only one cyst benign in structure. Our case was atypical to the normal presentation of mesotheliomas; however, the histology did confirm the diagnosis. Our patient was therefore completely treated by laparoscopic excision of the cyst. Nevertheless, follow-up is mandatory because of the possible recurrence of mesotheliomas.

\section{References}

1. Villaschi S, Autelitano F, Santeusanio G, Balistreri P (1990) Cystic mesothelioma of the peritoneum. A report of three cases. Am J antibodies. Clin Pathol 94:758-761

2. Mennemeyer R, Smith M (1979) Multicystic, peritoneal mesothelioma: a report with electron microscopy of a case mimicking intraabdominal cystic hygroma (lymphangioma). Cancer 44(1): 692-698

3. Pelosi G, Zannoni M, Caprioli F et al (1991) Peritoneal benign multicystic mesothelioma: a case report and review of the literature. Histl Histopatol 64:575-583

4. Asensio JA, Goldblatt P, Thomford NR (1990) Primary malignant peritoneal mesothelioma. A report of seven cases and a review of the literature. Arch Surg 125:1477-1481

5. Weiss SW, Tavassoli FA (1988) Multicystic mesothelioma: an analysis of pathologic findings and biologic behaviour in 37 cases. Am J Surg Pathol 12:737-746

6. O’Neil JD, Ros PR, Storm BL, Buck JL, Wilkinson EJ (1989) Cystic mesothelioma of the peritoneum. Radiology 170:333-337

7. Baddoura FK, Varma VA (1990) Cytological findings in multicystic peritoneal mesothelioma. Acta Cytol 34(4):524-528

8. Usha Kiran TS, Agboola A, Davies R, Stout TV (2002) Benign cystic mesothelioma: a diagnostic dilemma. Aust NZ J Obstet Gynaecol 42(5):552-554

9. Van Ruth S, Bronkhorst MW, Van Coevorden F, Zoetmulder FA (2002) Peritoneal benign cystic mesothelioma: a case report and review of the literature. Eur J Surg Oncol 28:192-195

10. Bui-Mansfield LT, Kim-Ahn G, O'Bryant L (2002) Multicystic mesothelioma of the peritoneum. AJR 178:402

11. Ordonez NG (2006) The diagnostic utility of immunohistochemistry and electron microscopy in distinguishing between peritoneal mesotheliomas and serous carcinomas: a comparative study. Mod Pathol 19(1):34-48 